\title{
Sensitividad Ansiosa y Afecto Negativo: Modelo transdiagnóstico para síntomas ansiosos y depresivos
}

\author{
Ronald Alberto Toro Tobar ${ }^{1}$, Sandra Milena Barrera ${ }^{2}$, \\ Betty Rocío Ramos Rodríguez ${ }^{3}$, Ana Isabel Velásquez Gordillo ${ }^{4}$ \\ Universidad Católica de Colombia
}

El objetivo del presente estudio fue identificar el papel predictivo de la sensitividad ansiosa (SA) y el afecto negativo (AN) de los síntomas ansiosos y depresivos, relación que no ha sido analizada con detenimiento. Se realizó un estudio correlacional multivariado con ecuaciones estructurales en un diagrama de senderos. La muestra fue no probabilística de 506 participantes $(M=31.96, D E=11.26$ años $)$. En los resultados se reportaron correlaciones significativas entre las variables transdiagnósticas y los síntomas, con diferencias amplias una vez controlado el AN. Los coeficientes de regresión jerárquica evidenciaron alta capacidad predictiva de la SA y el AN, en especial con los síntomas ansiosos. En un modelo estructural se presentó un ajuste favorable, sin evidencias de mediación del AN en la relación SA y síntomas. Se discutieron las posibilidades de un AN y SA como variables de segundo orden que sugieren un modelo transdiagnóstico de orden superior.

Palabras clave: depresión, ansiedad, sensitividad ansiosa, afecto negativo, transdiagnóstico.

\section{Anxiety Sensitivity and Negative Affect: Transdiagnostic model for anxious and depres- sive symptoms}

The aim of the study was to identify the predictive role of anxiety sensitivity (SA) and negative affect (AN) on anxiety and depression, a relationship that has not been analyzed in detail. A multivariate correlational study was carried out with structural equations. The sample included 506 participants $(M=31.96, S D=11.26$ years). Significant correlations were reported between the transdiagnostic variables and the symptoms, with wide differences once the $\mathrm{AN}$ was controlled. The regression coefficients showed a high predictive capacity of SA and AN, especially with anxiety. In a structural model, a favorable adjustment without evidence of AN mediation in the SA and symptoms. The possibilities of AN and SA are discussed as second-order variables that suggest a higher order model.

Keywords: depression, anxiety, anxiety sensitivity, negative affect, transdiagnostic.

$1 \quad \mathrm{PhD}(\mathrm{c})$ en Salud, Psicología y Psiquiatría (U. de Almería, España). Docente universitario. Dirección postal: Avenida Caracas \# 46 - 22, Bogotá, Colombia. Contacto: ratoro@ucatolica. edu.co. https://orcid.org/0000-0001-6061-3499

2 Psicóloga. Especialista en Psicología Clínica. Psicoterapeuta y orientadora en una institución de educación media. Dirección postal: Avenida Caracas \# 46 - 22, Bogotá, Colombia. Contacto: smbarrera@ucatolica.edu.co. https://orcid.org/0000-0002-5022-5019

3 Psicóloga. Especialista en Psicología Clínica. Investigadora criminológica en una empresa estatal. Contacto: brramos14@ucatolica.edu.co. https://orcid.org/0000-0003-0890-1287

4 Psicóloga. Especialista en Psicología Clínica. Coordinadora y psicoterapeuta en una institución prestadora de salud. Contacto: aivelasquez08@ucatolica.edu.co. https://orcid. org/0000-0002-3663-1643 


\section{Sensibilidade à Ansiedade e Afeto Negativo: Modelo de Transdiagnostico para sintomas ansiosos e depressivos}

O objetivo foi identificar o papel preditivo da sensibilidade ansiosa (SA) e afeto negativo (AN) nos sintomas de ansiedade e depressão, relação que não tem sido analisada em detalhe. Um estudo de correlação multivariado foi realizado com equaçóes estruturais em um diagrama de caminhos. A amostra foi não probabilística de 506 participantes $(M=31.96$, $D P=11.26$ anos). Nos resultados, correlaçóes significativas foram relatadas entre as variáveis transdiagnósticas e os sintomas, com grandes diferenças uma vez que o AN era controlado. Os coeficientes de regressão hierárquica mostraram alta capacidade preditiva de AS e AN, especialmente com sintomas de ansiedade. No modelo estrutural observa-se um ajuste favorável, sem evidência de mediação do $\mathrm{AN}$ na relação $\mathrm{SA}$ e sintomas. As possibilidades de $\mathrm{AN}$ e SA foram discutidas como variáveis de segunda ordem que sugerem um modelo transdiagnóstico de ordem superior.

Palavras-chave: depressão, ansiedade, sensibilidade ansiosa, afeto negativo, transdiagnóstico.

\section{Sensibilité à l'anxiété et effet négatif : Modèle transdiagnostique pour les symptômes anxieux et dépressifs}

L'objectif était d'identifier le rôle prédictif de la sensibilité l'anxiété (SA) et de l'affect négatif (AN) sur les symptômes de l'anxiété et de la dépression, relation qui n'a pas été analysée en détail. Une étude de corrélation multivariée a été réalisée avec des équations structurelles dans un diagramme de trajectoire. L'échantillon était non probabiliste sur 506 participants $(M=31.96, S D=11.26$ ans). Dans les résultats, des corrélations significatives ont été rapportées entre les variables transdiagnostiques et les symptômes, avec de grandes différences une fois que l'AN a été contrôlé. Les coefficients de régression hiérarchique ont montré une capacité prédictive élevée de l'AS et de l'AN, en particulier pour les symptômes d'anxiété. Dans un modèle structurel, un ajustement favorable a été présenté, sans évidence de médiation AN dans la relation et les symptômes de l'AS. Les possibilités de l'AN et de l'AS ont été discutées en tant que variables de second ordre suggérant un modèle transdiagnostique d'ordre supérieur.

Mots clés: dépression, anxiété, sensibilité à l'anxiété, affect négatif, transdiagnostic. 
Las cifras de salud mental de la Organización Mundial de la Salud (OMS) y la Organización Panamericana de Salud (PAHO), alertaron en el 2017 sobre los trastornos de ansiedad y depresión como los más frecuentes en la población latinoamericana. Resaltaron que la mayor prevalencia estaba en los trastornos de ansiedad que varían entre el $2.4 \%$ al $18.2 \%$. Siendo los más frecuentes en los últimos 12 meses la fobia específica con un $2.7 \%$ hasta un $8.7 \%$ ( $6 \%$ en Colombia), el trastorno depresivo mayor con un $1.5 \%$ hasta un $6.7 \%$ (5.6\% en Colombia), y la fobia social con un $1 \%$ hasta un $6.8 \%$ (2.5\% en Colombia). Asimismo, indicaron que la depresión se ha convertido en la principal causa de los problemas de salud y la discapacidad en todo el mundo, según la OPS/PAHO (2017), más de 300 millones de personas viven con depresión, con un incremento del 18\% entre los años 2005 al 2015. Es así que el abordaje de los trastornos mentales emocionales y afectivos, se convierten en una prioridad actual, los esfuerzos enfocados hacia la prevención han brindado direcciones hacia el desarrollo de modelos integrales que abarcan diferentes patologías y explicaciones acerca de los mecanismos causales comunes entre las mismas.

Uno de ellos ha sido el modelo transdiagnóstico, el cual ha permitido generar un marco de referencia con una conceptualización flexible que facilita la comprensión de los patrones de comorbilidad presentes en la psicopatología, el cual está basado en las variables comunes a nivel causal y de mantenimiento (Aldao, 2012). Al respecto, se han planteado diferentes modelos transdiagnósticos en las últimas décadas en el cual la investigación ha girado en torno a los síntomas compartidos, los múltiples diagnósticos, procesos y variables comunes a diferentes trastornos (Mansell, Harvey, Watkings \& Shafran, 2009).

Una de estas variables ha sido el afecto positivo y negativo, se trata de dos dimensiones que están relacionadas con las experiencias emocionales y las estructuras del afecto. El Afecto Positivo (AP) 
se caracteriza por una dimensión de emocionalidad positiva, energía, activación, afiliación, y predisposición a experimentar emociones positivas. En cambio, el Afecto Negativo (AN) está relacionado con estados de ánimos aversivos, ansiedad, hostilidad, inseguridad, temor, tristeza, y nerviosismo (Ambrona \& Lopez-Perez, 2014; Watson \& Tellegen, 1988). La combinación de ambos afectos daría cuenta de la correlación en los trastornos emocionales de ansiedad y depresión. Diferentes estudios han puesto en evidencia la correlación positiva que existe entre el afecto negativo y los síntomas de ansiedad y depresión (Guzmán, Padrós, García \& Laca, 2014; Zvolensky et al., 2016). Esto permitiría explicar el desarrollo y el mantenimiento de varios de los trastornos emocionales y afectivos en un modelo basado particularmente en el AN.

La Sensitividad Ansiosa (SA) es otra de las variables transdiagnósticas de amplio interés investigativo en la actualidad. La SA fue propuesta como un factor de predisposición para el desarrollo de los trastornos de ansiedad. Indica la tendencia de temor hacia las sensaciones relacionadas con la ansiedad, en el que se presentan como creencias sobre las sensaciones que llevan un riesgo para la persona; es decir, las consecuencias peligrosas o dañinas de los síntomas ansiosos (Reiss, 1991). Se ha reportado evidencia que la constituye como una variable de vulnerabilidad también hacia otros trastornos como los trastornos depresivos (Taylor, 1999), entre otros.

Según investigaciones recientes, este constructo se compone de una estructura jerárquica conformada por un factor general y tres dimensiones específicas: física, cognitiva y social (Farris et al., 2015). Estas dimensiones estarían relacionadas diferencialmente con los trastornos de ansiedad y depresión (Osma-López, García-Palacios, Botella-Arbona \& Barrada-González, 2014; Wheaton et al., 2012), y al parecer se constituye como un factor de riesgo clave para el desarrollo de estos trastornos.

Por ejemplo, se ha reportado que en pacientes con síntomas depresivos e ideación suicida se presentaron asociaciones significativas con la SA cognitiva (Norr, Allan, Macatee, Capron \& Schmidt, 2016). 
En otros estudios, se ha concluido que una elevada SA parece modular la relación entre ansiedad y los síntomas somáticos severos (v.g. Fergus, Kelley \& Griggs, 2016), lo cual predice incrementos en la conducta de tabaquismo como respuesta ante el malestar emocional y los estados de ansiedad relacionados (Leventhal \& Zolensky, 2015).

Es así que la SA parece estar relacionada con psicopatologías depresivas y ansiosas, e ideación suicida. Por ejemplo, en muestras latinas fueron reportados mayores síntomas asociados a la SA, dadas las condiciones particulares de esta población que presentan mayores niveles de AN y alta SA, con una clara tendencia a la depresión; sin embargo, la evidencia no es contundente con estas muestras en el momento (Zvolensky et al., 2016).

Estas conclusiones al parecer indican que la SA presenta un incremento en el AN, el cual estaría relacionado con un aumento en la intensidad de los síntomas propios de los trastornos ansiosos y depresivos. Se ha planteado que el AN funciona como posible modulador en la aparición de estos síntomas se ha reportado evidencia parcial en estudios sobre la disregulación emocional y el posible efecto indirecto del AN en el alcoholismo en adultos (Veilleux, Skinner, Reese \& Shaver, 2014). Aunque, la desregulación emocional también se ha considerado mediador entre el AN y la respuesta de craving en estos pacientes (Khosravani, Bastan, Ghorbani \& Kamali, 2017), aspecto que resalta el posible papel causal de la SA.

Según hallazgos para la ansiedad social, se ha sugerido que el AN puede modular negativamente la capacidad de reconocimiento de emociones en los rostros, un aspecto clave en los trastornos emocionales (Ball et al., 2012). A su vez, estaría indirectamente relacionado con la ansiedad social y la depresión a través de vulnerabilidades cognitivas, incluyendo otras variables como la intolerancia a la incertidumbre (Allan et al., 2017). Además de la SA, como medida de vulnerabilidad cognitiva entre la relación total o parcial que puede representar el AN en esta asociación, autores como Allan et al. (2017), sugieren desarrollar modelos con estructuras de orden superior para la SA. 
Estos estudios previos, dan cuenta que aún no está clara la relación entre las variables transdiagnósticas como son el AN y la SA en los síntomas de los trastornos del estado del ánimo y ansiedad. Por lo tanto, el presente estudió buscó identificar el papel predictivo de la SA y el AN sobre los síntomas del espectro ansioso y depresivo en un modelo transdiagnóstico.

\section{Método}

\section{Participantes}

La técnica de muestreo utilizada para esta investigación fue un muestreo no probabilístico por conveniencia incidental. Los participantes se seleccionaron teniendo en cuenta los siguientes criterios de inclusión: presentar edades que oscilaran entre los 18 años y los 65 años, participar voluntariamente y firmar el consentimiento informado; se excluyeron aquellas personas con patologías diferentes a las emocionales y afectivas. La muestra final estuvo conformada por 506 participantes colombianos voluntarios entre 18 y 64 ańos de edad, con una media de $31.96(D E=11.26)$, el $37.2 \%$ fueron hombres $(n=188)$ y el $62.6 \%$ mujeres $(n=317)$, un $51.6 \%$ solteros, un $22.7 \%$ casados, y un $22.3 \%$ en unión libre, de los cuales el $27.5 \%$ contaba con una escolaridad secundaria, un $18.6 \%$ a nivel técnico y un $39.7 \%$ con educación superior.

Teniendo en cuenta la ley colombiana 1090 de 2006, en la que se reglamenta el ejercicio de la profesión de psicología, el psicólogo debe abordar la investigación respetando la dignidad y el bienestar de las personas que participan y con pleno conocimiento de las normas legales y de los estándares profesionales que regulan la conducta de investigación con participantes humanos. Por ello, se exigió la firma de un consentimiento informado a cada uno de los participantes, con el fin de garantizar cumplir los códigos de ética sobre la participación de los sujetos humanos en investigaciones con riesgo mínimo. 


\section{Medición}

Afecto positivo y negativo. Se utilizó la Escala de Afecto Positivo y Afecto Negativo - PANAS_, en su versión adaptada al idioma español por Robles y Páez (2003) de la original elaborada por Watson et al. (1988). Esta escala presenta en una serie de reactivos que describen los estados afectivos. Se compone de 40 ítems en dos apartados de veinte ítems cada uno, diez miden el afecto positivo y diez el negativo. En el primer apartado se evalúa la presencia de los afectos "en las últimas semanas" (afecto como estado), y en el segundo apartado se les evalúa "generalmente" (afecto como rasgo). Los sujetos contestan según una escala tipo Likert con un rango de 1 (muy poco o casi nada) hasta 5 (extremadamente). El PANAS se caracteriza por una alta consistencia interna, con coeficientes alfas de $\alpha=.86$ a $\alpha=.90$ para el afecto positivo, y de $\alpha=.84$ a $\alpha=.87$ para el afecto negativo, además de favorables indicadores de confiabilidad test-retest $(r=.71$ para rasgo y $r=.68$ para estado) como medidas de estabilidad del afecto (Robles \& Páez, 2003). En la muestra total del presente estudio, se obtuvieron adecuados indicadores de fiabilidad según los coeficientes alfa de Cronbach de $\alpha=.77$ para estado, y $\alpha=.81$ para rasgo.

Sensitividad Ansiosa. Se utilizó el Índice de Sensibilidad a la Ansiedad -ASI-3 - (Taylor et al., 2007; versión española de Sandín, Valiente, Chorot \& Santed, 2007), que es una escala compuesta por 18 ítems que se deben responder según una escala tipo Likert de 0 (Nada o casi nada) a 4 (Muchísimo). Estos ítems hacen referencia a reacciones de miedo o ansiedad ante la experiencia de síntomas de físicos, por ejemplo: "cuando siento opresión en el pecho, me asusta no poder respirar bien", síntomas cognitivos, por ejemplo: "cuando me resulta difícil pensar con claridad, me preocupa que me esté ocurriendo algo grave", y síntomas sociales, "Tengo miedo a sonrojarme delante de la gente". Cada subescala contiene seis ítems, la ASI-3 presenta una favorable consistencia interna para cada factor según el coeficiente Alpha de Cronbach $\alpha=.89, \alpha=.90$ y $\alpha=.85$. En la muestra total del presente estudio, se obtuvo un adecuado favorable indicador de fiabilidad alfa de Cronbach de $\alpha=.92$. 
Síntomas ansiosos. Se utilizó el Inventario de Ansiedad de BeckBAI-(Beck, Epstein, Brown \& Steer, 1988), el cual es un instrumento que evalúa la presencia e intensidad de los síntomas de ansiedad fisiológicos y cognitivos. Se compone de 21 ítems que evalúan la gravedad de la sintomatología ansiosa según cuatro alternativas ordenadas de menor a mayor gravedad del estado ansioso durante las últimas dos semanas, en una escala que va de 0 a 3 puntos. La adaptación española elaborada por Sanz, García-Vera y Fortun (2012) presentó un $\alpha=.90$ para muestras españolas, una puntuación media en pacientes con ansiedad de $25.7(D E=11.4)$ y para no ansiosos una media de $15.8(D E=11.8)$. En la muestra total del presente estudio, se obtuvo un adecuado indicador de fiabilidad según el coeficiente alfa de Cronbach de $\alpha=.92$.

Síntomas depresivos. Se utilizó el Inventario de Depresión de Beck -BDI-II- (Beck, Steer \& Brown, 1996), el cual fue diseñado para evaluar la intensidad de la sintomatología depresiva, es auto aplicable y consta de 21 reactivos con cuatro opciones de respuesta que representan el espectro de gravedad sintomática evaluada. Un valor cero indica ausencia del síntoma y tres una gravedad máxima del síntoma. Este instrumento fue adaptado al español por Brenlla y Rodríguez (2006), presenta un indicador de consistencia interna favorable con un coeficiente de Cronbach de $\alpha=.88$ en pacientes con diagnóstico previo de depresión y un $\alpha=.94$ para población sin diagnóstico. En la muestra total del presente estudio, se obtuvo un adecuado indicador de fiabilidad según el coeficiente alfa de Cronbach de $\alpha=.92$.

\section{Procedimiento}

En primer lugar, se llevó a cabo la conformación de la batería de instrumentos en un único paquete de pruebas psicométricas. Luego, se convocó a la población a participar en la aplicación en un periodo de dos meses (junio y julio de 2017). Una vez fueron abordados los participantes, se le solicitó a cada uno de ellos que firmara el consentimiento informado asegurando su privacidad y la confidencialidad, teniendo en cuenta los parámetros éticos y legales para su participación en la investigación. Posteriormente, se realizó la tabulación de los datos en el 
programa de Microsoft Excel y consecutivamente se realizó el análisis estadístico final.

\section{Análisis de datos}

Se utilizó el paquete SPSS-25 y su extensión AMOS-23 para ejecutar los análisis estadísticos del estudio. Se realizó un análisis descriptivo de los datos calculando las medidas de tendencia central (media, desviación estándar, rango), y la distribución en cuanto a la asimetría y curtosis. Posteriormente, se llevó a cabo un análisis de correlación paramétrica con el $r$ de Pearson (orden cero y parcial), unos análisis de regresión múltiple con pasos sucesivos y un modelo estructural con un diagrama de senderos, mediantes indicadores de ajuste para ecuaciones estructurales y la revisión de los efectos directos e indirectos de cada variable transdiagnóstica.

\section{Resultados}

La puntuación obtenida en cada uno de los instrumentos utilizados en la aplicación, se presentan en la Tabla 1 . Se puede observar que los indicadores de dispersión y tendencia central fueron favorables para el posterior análisis estadístico multivariado.

Las correlaciones de Pearson de orden cero con un nivel de significancia $p>.01$ se presentan en la Tabla 2. Dado el modelo transdiagnóstico, se encontraron elevadas asociaciones entre el AN y la SA según las variables SA con el AN estado. De igual forma, se obtuvo un $r$ $=.44(p>.01)$ y $r=.45(p>.01)$ con el AN rasgo. Asimismo, una alta $y$ significativa asociación con depresión $(r=.41, p>.01)$, y mucho mayor con la variable sintomática de ansiedad $(r=.57, p>.01)$ siendo esta última mucho más representativa según las hipótesis del estudio. A su vez, se resaltan las bajas correlaciones entre la SA física y el AN estado $(r=.27 ; p>.01)$, y la elevada correlación entre la SA cognitiva con los síntomas de ansiedad $(r=.54 ; p>.01)$. 


\section{Tabla 1}

Estadísticos descriptivos obtenidos de los instrumentos utilizados

\begin{tabular}{|c|c|c|c|c|c|c|c|c|}
\hline \multicolumn{2}{|c|}{ Test / Variable } & Categorías & $M$ & $D E$ & Mín. & Máx. & Asimetría & Curtosis \\
\hline \multirow{4}{*}{ ASI-3 } & \multirow{4}{*}{$\begin{array}{l}\text { Sensiti- } \\
\text { vidad } \\
\text { Ansiosa }\end{array}$} & Cognitiva & 4.71 & 4.73 & 0 & 22 & 1.23 & 1.26 \\
\hline & & Social & 7.50 & 5.26 & 0 & 24 & 0.72 & 0.00 \\
\hline & & Física & 6.70 & 5.62 & 0 & 24 & 0.81 & 0.22 \\
\hline & & Total & 18.91 & 13.61 & 0 & 63 & 0.81 & 0.23 \\
\hline \multirow{6}{*}{ PANAS } & \multirow{3}{*}{$\begin{array}{l}\text { Afecto } \\
\text { (Rasgo) }\end{array}$} & A. Positivo & 34.52 & 8.00 & 10 & 57 & -0.47 & 0.13 \\
\hline & & A. Negativo & 18.43 & 6.92 & 10 & 44 & 1.10 & 1.00 \\
\hline & & Total Rasgo & 52.94 & 9.81 & 21 & 85 & -0.00 & 0.25 \\
\hline & \multirow{3}{*}{$\begin{array}{l}\text { Afecto } \\
\text { (Estado) }\end{array}$} & A. Positivo & 34.03 & 7.91 & 10 & 50 & -0.40 & -0.27 \\
\hline & & Negativo & 18.70 & 6.96 & 10 & 45 & 1.13 & 0.96 \\
\hline & & Total Estado & 0.42 & 9.54 & 25 & 84 & 0.19 & 0.35 \\
\hline BAI & Ansiedad & Total & 12.29 & 10.84 & 0 & 54 & 1.13 & 1.19 \\
\hline BDI-II & Depresión & Total & 8.59 & 8.72 & 0 & 55 & 1.53 & 3.10 \\
\hline
\end{tabular}

Nota: $M$ (Media), DE (Desviación Estándar), ASI-3(Índice de Sensibilidad a la Ansiedad), PANAS (Escalas del Afecto Negativo estado y rasgo), BAI (Inventario de Ansiedad de Beck), BDI-II (Inventario de Depresión de Beck, segunda edición).

\section{Tabla 2}

Correlaciones de Pearson de orden cero para sensitividad ansiosa, afecto negativo, ansiedad y depresión $(n=506)$

\begin{tabular}{lccccccc}
\hline Variable & 1 & 2 & 3 & 4 & 5 & 6 & 7 \\
\hline 1. ASI-3 & - & & & & & \\
2. SC & $.87^{* *}$ & - & & & & \\
3. SS & $.87^{* *}$ & $.66^{* *}$ & - & & & \\
4. SF & $.87^{* *}$ & $.69^{* *}$ & $.59^{* *}$ & - & & \\
5. ANe & $.44^{* *}$ & $.41^{* *}$ & $.47^{* *}$ & $.28^{* *}$ & - & & \\
6. ANr & $.46^{* *}$ & $.43^{* *}$ & $.49^{* *}$ & $.27^{* *}$ & $.82^{* *}$ & - & \\
7. BDI-II & $.41^{* *}$ & $.38^{* *}$ & $.39^{*}$ & $.31^{* *}$ & $.51^{* *}$ & $.49^{* *}$ & - \\
8. BAI & $.57^{* *}$ & $.54^{* *}$ & $.52^{* *}$ & $.46^{* *}$ & $.56^{* *}$ & $.51^{* *}$ & $.53^{* *}$ \\
\hline
\end{tabular}

Nota: ASI-3 (Sensitividad Ansiosa), SC (Sensitividad Cognitiva), SS (Sensitividad Social), SF (Sensitividad Física), ANE (Afecto Negativo Estado), ANr (Afecto Negativo Rasgo), BDI-II (Inventario de Depresión de Beck, segunda edición), BAI (Inventario de Ansiedad de Beck), ${ }^{* *} p<.01$ (bilateral). ${ }^{*} p<.05$ (bilateral). 
A continuación, se revisó si al controlar las variables AN estado y rasgo, las correlaciones entre las variables sintomáticas y la SA cambiarían significativamente. En la Tabla 3, aparecen las correlaciones parciales ejecutadas en el que se puede observar que se obtuvo una correlación significativa al controlar el AN estado y AN rasgo; sin embargo, se evidenció un decremento al cambiar a un $r=.24$ para depresión; en los síntomas de ansiedad, al controlar el AN estado y AN rasgo en la correlación con la SA, se disminuyó a un $r=.44(p>.01)$, valores que representan una disminución significativa con respecto a los indicadores de correlación de orden cero.

\section{Tabla 3}

Correlaciones parciales y regresiones entre ansiedad, depresión y sensitividad ansiosa, controlado para afecto negativo estado-rasgo $(n=506)$

\begin{tabular}{lccccc}
\hline Correlación & $\begin{array}{c}\text { Variable } \\
\text { controlada }\end{array}$ & $\begin{array}{c}\text { Pearson's } r \\
\text { (parcial) }\end{array}$ & $A R^{2}$ & $B$ & IC 95\% \\
\hline Depresión*SA & ANE & $.24^{* *}$ & .35 & $.56^{* *}$ & .47 y .66 \\
& $\mathrm{ANr}$ & $.24^{* *}$ & .32 & $.53^{* *}$ & $.43 \mathrm{y} .63$ \\
Ansiedad*SA & $\mathrm{ANE}$ & $.44^{* *}$ & .44 & $.63^{* *}$ & $.52 \mathrm{y} .74$ \\
& $\mathrm{ANr}$ & $.44^{* *}$ & .40 & $.53^{* *}$ & $.41 \mathrm{y} .65$ \\
\hline
\end{tabular}

Nota: SA(Sensitividad Ansiosa), ANest (Afecto Negativo Estado), ANrasg (Afecto Negativo Rasgo). ${ }^{* *}$. $p<.01$ (bilateral). ${ }^{*} . p<.05$ (bilateral).

Los coeficientes de regresión y valores $\beta$ indicaron asociaciones significativas entre las variables predictoras y criterio, con diferencias entre los valores obtenidos luego de haber sido controlado el AN estado y rasgo, esto sugiere efectos indirectos significativos para depresión y menores en ansiedad. Se procedió a validar esta hipótesis usando un modelo de senderos (path analysis) en el que las dimensiones de la SA (física, cognitiva y social), predicen los síntomas de ansiedad con un $49 \%$ de la varianza total, y en depresión en menor medida con un 33\% de la varianza (ver Figura 1). 


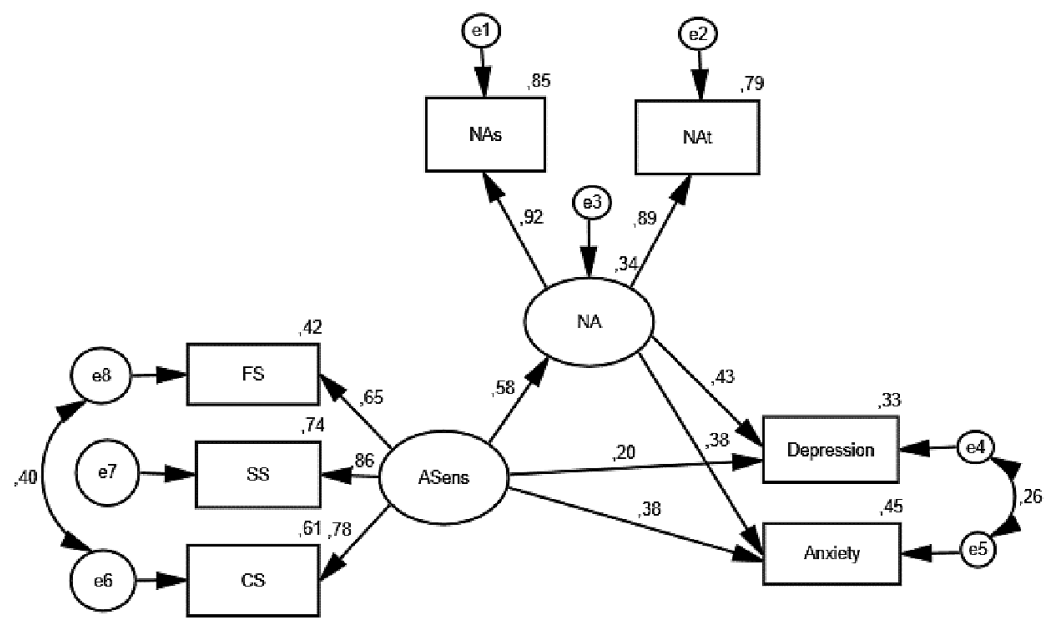

Figura 1. Modelo de senderos resultados basados en las capacidades predictivas de las variables $S A$ y $A N$, establecidas teóricamente para ansiedad $y$ depresión.

Nota: FS(síntomas físicos), SS(síntomas sociales), CS(síntomas cognitivos), ASens(sensitividad ansiosa), NA(afecto negativo), NAs(afecto negativo estado), NAt(afecto negativo rasgo).

En el modelo, según el método de máxima verosimilitud, a través de ecuaciones estructurales, se encontró que el ajuste según los indicadores $\chi^{2} / g l=4.19$ resultó favorable al estar por debajo de 5. Asimismo, en los valores de bondad de ajuste en este estudio se obtuvieron valores $G F I=.98$ (Goodness of Fit Index), TLI = .97 (Tucker Lewis Index), y $C F I=.98$ (Comparative Fit Index), deben ser superiores a .90 para ser considerados indicadores de buen ajuste. De igual forma, el ajuste completo del modelo representado con la mínima diferencia con respecto al valor poblacional estimado, también resultaron favorables según el $R M S E A=.07$ (Root Mean Square Error Aproximation, el valor sugerido es menor a .08), y el $R M R=.018$ (Root Mean Square Residual, el valor esperado es cercano a 0) (Hu \& Bentler, 1998).

Posteriormente, se revisaron los efectos directos e indirectos que se presentaban en el modelo estructural resultante según las variables predictoras SA y AN. Se encontró que los efectos directos de la SA 
$(D E=1)$ sobre ansiedad presentaba una $D E=.45$, mientras que ante $A N$, la variación en ansiedad fue $D E=.33$. En depresión según la $S A$ el efecto fue menor $(D E=.22)$, y ante el $A N$ una $D E=.42$. Estos valores directos indican que el AN puede representar una mayor capacidad explicativa que la SA en un modelo mediacional estructural. En los efectos indirectos, en ansiedad la SA variaba $D E=.19$, y el AN variaba $D E=.00$. En depresión, la $S A$ variaba .25 y $D E=.00$ ante el AN. Estos valores indican que el modelo mediacional total o parcial del AN no resultó estadísticamente significativo en un modelo de senderos con ecuaciones estructurales (enfoque SEM) (Ato \& Vallejo, 2011).

\section{Discusión}

El propósito del presente estudio fue identificar el papel predictivo de la SA y el AN sobre los síntomas del espectro ansioso y depresivo en un modelo transdiagnóstico. Los valores asociativos entre estas dos variables permitieron aseverar que el propósito fue cumplido de forma parcial, ya que los valores de regresión y mediación no fueron significativos para comprobar una hipótesis mediacional del AN en la aparición de los síntomas. Los valores predictivos parecen indicar que la SA y el AN incrementan los síntomas ansiosos y en menor medida los depresivos.

Estos hallazgos dan cuenta del AN como variable transdiagnóstica común a diferentes trastornos emocionales, al parecer se trata de una variable de orden superior que se constituye como un importante factor etiológico (Clark \& Watson, 1991), identificado de forma transversal en estudios realizados con diferentes trastornos de ansiedad y depresión, incluso en reportes de protocolos de intervención (Talkovsky \& Norton, 2014; Watson, Clark \& Stasik, 2011). El ANE resultó con mayores indicadores de asociación con la SA y los síntomas ansiosos y depresivos. Aunque, la diferencia fue baja con respecto al ANR, coincide con los reportes de diferentes autores quienes sugieren que esta última variable se constituye como un factor estable de malestar, tal como es el neuroticismo dado su elevado componente vegetativo, 
especialmente en las respuestas de ansiedad (Paulus, Talkovsky, Heggeness \& Norton, 2015).

Asimismo, la SA más el AN presentaron una significativa varianza explicada de los problemas emocionales y afectivos, en especial en ansiedad (49\%), estos datos concuerdan con los reportados por Paulus et al. (2015), los cuales presentaron un modelo que incluía un factor de orden superior para el AN teniendo en cuenta la SA y la intolerancia a la incertidumbre en su modelo resultante.

Sin embargo, los datos son inconclusos aún, dado que la capacidad mediadora del AN no resultó significativa y, por lo tanto, los predictores más robustos podrían estar centrados directamente en la SA, en particular para los síntomas de ansiedad. Esto coincide con los hallazgos reportados en estudios que evaluaron la SA en un amplio rango de trastornos (Ino et al., 2017), en especial en la gravedad de los trastornos de pánico (OsmaLópez, Barrada-González, García-Palacios \& Botella-Arbona, 2016).

Es necesario ahondar esta diferencia entre ambas variables transdiagnósticas en cuanto a su relación causal con los trastornos ansiosos y depresivos. Dado que existen reportes de su capacidad predictiva de los síntomas y su gravedad, en particular si se asocian a otras variables transdiagnósticas como la intolerancia a la incertidumbre. Esta variable ha mostrado también una elevada capacidad predictiva, pese a sus escasas especificidades para los trastornos (Hong \& Cheung, 2014). Es así que algunos autores indican que la SA media la relación entre AN y los síntomas internalizantes en participantes deportistas (BromanFulks, Abraham, Thomas, Canu \& Nieman, 2018), aspecto que podría plantear un modelo diferente al ajustado en el presente estudio en el que la SA tiene un elevado coeficiente de regresión de las variables sintomáticas ( $A R^{2}$ entre .35 y .44 para estado y rasgo).

Aclarar estos puntos puede generar implicaciones clínicas positivas en la comprensión de los factores causales de los trastornos emocionales y afectivos. La SA se considera un factor cognitivo afectivo clave de vulnerabilidad en trastornos como el trastorno por estrés postraumático y el abuso de sustancias, en particular porque la SA puede mediar esta relación (Berenz et al., 2016; Leventhal \& Zvolensky, 2015). 
Sin embargo, como respuesta ante el craving el papel del AN puede resultar un factor moderador que no se ha tenido en cuenta (Vujanovic et al., 2018). Sería importante indagar con mayor escrutinio el papel de estas variables en otros espectros de la psicopatología como el abuso de sustancias y los trastornos de la conducta alimentaria.

El presente estudio contó con algunas limitaciones por reconocer. En primer lugar, pese a que los indicadores de ajuste del modelo fueron favorables, se identificó que el uso de variables como la intolerancia a la incertidumbre pudo generar mejores índices, lo cual estaría en consonancia con los hallazgos de autores como Paulus et al. (2015).

En segundo lugar, estas hipótesis deben ser comprobadas con muestras clínicas con características propias de los trastornos emocionales y afectivos, preferiblemente, teniendo en cuenta los diagnósticos previos, en especial aquellos trastornos que tengan evidencia de la causalidad de las variables como las usadas en el estudio. Esto permitiría comprobar modelos basados en las diferencias entre grupos sintomáticos y los cambios posibles entre cada patología y la comorbilidad que presentan.

En tercer lugar, el uso de las escalas adaptadas al idioma español y la región favorecería la precisión de las medidas usadas en el estudio. Sin embargo, es necesario contar con mayores análisis de sus capacidades predictivas, validez convergente con los síntomas y medidas equivalentes ya conocidas. Asimismo, el análisis de invarianzas entre grupos caracterizados por el sexo o grupos patológicos aportaría al panorama de investigación clínica que sigue en permanente desarrollo en la actualidad.

Para finalizar, a manera de conclusión se puede afirmar que la variable transdiagnóstica SA presentó una elevada capacidad predictiva de los síntomas de ansiedad y depresión: mientras que el AN como otra variable transdiagnóstica, incluida en un modelo de senderos, incrementa también la aparición de los síntomas emocionales y afectivos. Esto ocurre particularmente con los relacionados a los trastornos de ansiedad. Sin embargo, es necesario seguir indagando la capacidad predictiva de estas dos variables en un modelo transdiagnóstico que incluya factores de orden superior y otras variables ya reconocidas por su evidencia en la actualidad de la psicología clínica. 


\section{Referencias}

Aldao, A. (2012). Emotion regulation strategies as transdiagnostic processes: A closer look at the invariance of their form and function. Revista de Psicopatología y Psicología Clinica, 17(3), 261277. https://doi.org/10.5944/rppc.vol.17.num.3.2012.11843

Ambrona, T. \& López-Pérez, B. (2014). A Longitudinal Analysis of the Relationship between Positive and Negative Affect and Health. Psychology, 5, 859-863. https://doi.org/10.4236/ psych.2014.58097

Allan, P., Cooper, D., Oglesby, M., Short, N., Saulnier, G. \& Schmidt, B. (2017). Lower-order anxiety sensitivity and intolerance of uncertainty dimensions operate as specific vulnerabilities for social anxiety and depression within a hierarchical mode, Journal of Anxiety Disorders, 53, 91-99. https://doi.org/10.1016/j. janxdis.2017.08.002

Ato, M. \& Vallejo, G. (2011). Los efectos de terceras variables en la investigación psicológica. Anales de Psicología, 27(2), 550-561. Disponible en http://revistas.um.es/analesps

Ball T. M., Sullivan, S., Flagan, T., Hitchcocka, C. A., Simmons, A., Paulus, M. P. \& Stein, M. B. (2012). Selective effects of social anxiety, anxiety sensitivity, and negative affectivity on the neural bases of emotional face processing. NeuroImage, 59(2), 18791887. https://doi.org/10.1016/j.neuroimage.2011.08.074

Beck, A. T., Epstein, N., Brown, G. \& Steer, R. A. (1988). An inventory for measuring clinical anxiety: psychometric properties. Journal of Consulting and Clinical Psychology, 56(6), 893-897. https:// doi.org/10.1037/0022-006X.56.6.893

Beck, A. T., Steer, R. A. \& Brown, G. K. (1996). Manual for the Beck Depression Inventory-II. Texas, TX: Psychological Corporation. https://doi.org/10.1037/t00742-000

Berenz, E. C., Kevorkian, S., Chowdhury, N., Dick, D. M., Kendler, K. S. \& Amstadter, A. B. (2016). Posttraumatic stress disorder symptoms, anxiety sensitivity, and alcohol use motives in college 
students with a history of interpersonal trauma. Psychology of Addictive Behaviors, 30(7), 755-763. https://doi.org/10.1037/ adb0000193

Brenlla, M. \& Rodríguez, C. (2006). Beck BDI-II Depression Inventory Manual Argentine Adaptation. Buenos Aires: Paidós.

Broman-Fulks, J. J., Abraham, C. M., Thomas, K., Canu, W. H. \& Nieman, D. C. (2018). Anxiety sensitivity mediates the relationship between exercise frequency and anxiety and depression symptomology. Stress and Health, 34, 500-508. https://doi.org/10.1002/smi.2810

Clark, L. A. \& Watson, D. (1991). Tripartite model of anxiety and depression: Psychometric evidence and taxonomic implications. Journal of Abnormal Psychology, 100, 316. https://doi. org/10.1037/0021-843X.100.3.316

Farris, S. G., DiBello, A. M., Allan, N. P., Hogan, J., Schmidt, N. B. \& Zvolensky, M. J. (2015). Evaluation of the Anxiety Sensitivity Index-3 among treatment-seeking smokers. Psychological Assessment, 27(3), 1123-128. https://doi.org/10.1037/pas 0000112

Fergus, T. A., Kelley, L. P. \& Griggs, J. O. (2016). The combination of health anxiety and somatic symptoms: Specificity to anxiety sensitivity cognitive concerns among patients in primary care. Psychiatry Research, 239, 117-121. https://doi.org/10.1016/j. psychres.2016.02.067

Hong, R. Y. \& Cheung, M. W.-L. (2014). The structure of cognitive vulnerabilities to depression and anxiety evidence for a common core etiologic process based on a meta-analytic review. Clinical Psychological Science, 3(6), 892-912. https://doi. org/10.1177/2167702614553789

Hu, L. \& Bentler, P. M. (1988). Fit indices in covariance structure modeling: Sensitivity to underparameterized model misspecification. Psychological Methods, 3(4), 424-453. https:// doi.org/10.1037/1082-989X.3.4.424 
Ino, K., Ogawa, S., Kondo, M., Imai, R., Ii, T., Furukawa, T. A. \& Akechi, T. (2017). Anxiety sensitivity as a predictor of broad dimensions of psychopathology after cognitive behavioral therapy for panic disorder. Neuropsychiatric Disease and Treatment, 13, 1835-1840. https://doi.org/10.2147/NDT.S121360

Khosravani, V., Bastan, F. S., Ghorbani, F. \& Kamali, Z. (2017). Difficulties in emotion regulation mediate negative and positive affects and craving in alcoholic patients. Addictive Behaviors, 71, 75-81. https://doi.org/10.1016/j.addbeh.2017.02.029

Leventhal, A. M. \& Zvolensky, M. J. (2015). Anxiety, depression, and cigarette smoking: A transdiagnostic vulnerability framework to understanding emotion-smoking comorbidity. Psychological Bulletin, 141(1), 176-212. https://doi.org/10.1037/bul0000003

Mansell, W., Harvey, A., Watkings, E. \& Shafran, R. (2009). Conceptual foundations of the transdiagnostic approach to CBT. Journal of Cognitive Psychotherapy: An International Quarterly, 23(1), 6-19. https://doi.org/10.1891/0889-8391.23.1.6

Norr, A. M., Allan, N. P., Macatee. R. J., Capron, R. J. \& Schmidt, B. N. (2016). The role of anxiety sensitivity cognitive concerns in suicidal ideation: A test of the Depression-Distress Amplification Model in clinical outpatients. Psychiatry Research, 238, 74-80. https://doi.org/10.1016/j.psychres.2016.02.016

Organización Panamericana de Salud \& Organización Mundial de la Salud (2017). Depresión: Hablemos", dice la OMS, mientras la depresión encabeza la lista de causas de enfermedad. Recuperado de https://www.paho.org/uru/index.php?option=com_content \&view=article\&id=1152:depresion-hablemos-dice-la-omsmientras-la-depresion-encabeza-la-lista-de-causas-de-enfermedad \&Itemid $=340$

Osma-López, J., Barrada-González, J. R., García-Palacios, A. \& Botella-Arbona, C. (2016). Influence of vulnerability factors in panic disorder severity. Psicothema, 28(2), 167-173. https://doi. org/10.7334/psicothema2015.223 
Osma-López, J., García-Palacios, A., Botella-Arbona, C. \& BarradaGonzález, J. R. (2014). Personalidad y sensibilidad a la ansiedad como factores de vulnerabilidad en el trastorno de pánico. Psicología Conductual, 22(2), 185-201.

Paulus, D. J., Talkovsky, A. M., Heggeness, L. F. \& Norton, P. J. (2015). Beyond negative affectivity: a hierarchical model of global and transdiagnostic vulnerabilities for emotional disorders. Cognitive Behaviour Therapy, 44(5), 389-405. https://doi.org/10.1080/16 506073.2015.1017529

Reiss, S. \& McNally, R. J. (1985). Expectancy model of fear. En S. Reiss y R. R. Bootzin (Eds.), Theoretical issues in behavior therapy (pp. 107-121). San Diego, CA: Academic Press.

Robles, R. \& Páez, F. (2003). Estudio sobre la traducción al español y las propiedades psicométricas de las escalas de afecto positivo $y$ negativo (panas). Salud Mental, 26, 69-75.

Sandín, B., Valiente, R. M., Chorot, P. \& Santed, M. A. (2007). ASI-3: Nueva escala para la evaluación de la sensibilidad a la ansiedad. Revista de Psicopatología y Psicología Clínica, 12(2), 91-104. https://doi.org/10.5944/rppc.vol.12.num.2.2007.4036

Sanz, J., García-Vera, M. \& Fortun, M. (2012). The "Beck Anxiety Inventory" (BAI): Psychometric properties of the spanish version in patients with psychological disorders. Behavioral Psychology/ Psicología Conductual, 20(3), 563-583.

Talkovsky, A. \& Norton, P. (2014). Mediators of transdiagnostic group cognitive behavior therapy. Journal of Anxiety Disorders, 28(8), 919-924. https://doi.org/10.1016/j.janxdis.2014.09.017

Taylor, S. (1999). Anxiety sensitivity: Theory, research, and treatment of the fear of anxiety. Mahwah, NJ: Erlbaum.

Veilleux, J.C., Skinner, K.D., Reese, E. D. \& Shaver, J.A. (2014). Negative affect intensity influences drinking to cope through facets of emotion dysregulation. Personality and Individual Differences, 59, 96-101. https://doi.org/10.1016/j. paid.2013.11.012 
Vujanovic, A. A., Farris, S. G., Bartlett, B. A., Lyons, R. C., Haller, M., Colvonen, P. J., Norman, S.B. (2018). Anxiety sensitivity in the association between posttraumatic stress and substance use disorders: A systematic review. Clinical Psychology Review, 62, 37-55. https://doi.org/10.1016/j.cpr.2018.05.003

Watson, D., Clark, L.A. \& Stasik, S. M. (2011). Emotions and the emotional disorders: A quantitative hierarchical perspective. International Journal of Clinical and Health Psychology, 11(3), 429442. Disponible en www.redalyc.org/pdf/337/33719289001.pdf Watson, D., Clark, L.A. \& Tellegen, A. (1988). Development and validation of brief measures of positive and negative affect: the PANAS scales. Review of Personality and Social Psychology, 54(6), 1063-1070. https://doi.org/10.1037/0022-3514.54.6.1063

Wheaton, M.G., Deacon, B.J., McGrath, P. B., Berman, N.C. \& Abramowitz, J.S. (2012). Dimensions of anxiety sensitivity in the anxiety disorders: evaluation of the ASI-3. Journal of Anxiety Disorders, 26, 401-408. https://doi.org/10.1016/j.janxdis.2012. 01.002

Zvolensk, M. J., Paulus, D. J., Bakhshaie, J., Garza, M., Ochoa-Perez, M., Medvedeva, A. \& Schmidt, NB. (2016). Interactive effect of negative affectivity and anxiety sensitivity in terms of mental health among Latinos in primary care. Psychiatry Research, 243, 35-42. https://doi.org/10.1016/j.psychres.2016.06.006

Recibido: 27 de julio, 2018

Revisado: 21 de enero, 2019 Aceptado: 18 de marzo, 2019 